\title{
Down-regulation of the potassium-chloride cotransporter KCC2 contributes to spasticity after spinal cord injury
}

\author{
Pascale Boulenguez ${ }^{1,7}$, Sylvie Liabeuf ${ }^{1,7}$, Rémi Bos ${ }^{1}$, Hélène Bras $^{1}$, Céline Jean-Xavier ${ }^{1,6}$, Cécile Brocard ${ }^{1}$, \\ Aurélie Stil ${ }^{1}$, Pascal Darbon ${ }^{1,6}$, Daniel Cattaert ${ }^{2}$, Eric Delpire ${ }^{3}$, Martin Marsala ${ }^{4,5}$ \& Laurent Vinay ${ }^{1}$
}

Hyperexcitability of spinal reflexes and reduced synaptic inhibition are commonly associated with spasticity after spinal cord injury $(\mathrm{SCl})$. In adults, the activation of $\gamma$-aminobutyric acid ${ }_{A}\left(\mathrm{GABA}_{A}\right)$ and glycine receptors inhibits neurons as a result of low intracellular chloride $\left(\mathrm{Cl}^{-}\right)$concentration, which is maintained by the potassium-chloride cotransporter KCC2 (encoded by SIC12a5). We show that KCC2 is downregulated after SCI in rats, particularly in motoneuron membranes, thereby depolarizing the $\mathrm{Cl}^{-}$equilibrium potential and reducing the strength of postsynaptic inhibition. Blocking KCC2 in intact rats reduces the rate-dependent depression (RDD) of the Hoffmann reflex, as is observed in spasticity. RDD is also decreased in KCC2deficient mice and in intact rats after intrathecal brain-derived neurotrophic factor (BDNF) injection, which downregulates KCC2. The early decrease in KCC2 after SCI is prevented by sequestering BDNF at the time of SCI. Conversely, after SCI, BDNF upregulates KCC2 and restores RDD. Our results open new perspectives for the development of therapeutic strategies to alleviate spasticity.

Spasticity is a disabling complication affecting individuals with $\mathrm{SCI}^{1}$. About $75 \%$ of individuals with SCI show spasticity 1 year after injury, and about half of them receive antispasticity medication. None of the currently available drugs is uniformly useful in lessening all of the symptoms of spasticity, and these drugs have potentially serious side effects. Spasticity is characterized by a velocitydependent increase in muscle tone resulting from hyperexcitable stretch reflexes, spasms and hypersensitivity to normally innocuous sensory stimulations. The main mechanisms hypothesized to be responsible for spasticity are increased motoneuron excitability ${ }^{2,3}$ and increased synaptic inputs in response to muscle stretch due to reduced inhibitory mechanisms (presynaptic ${ }^{4}$, recurrent ${ }^{5}$, reciprocal inhibitions ${ }^{6}$ and flexor reflex afferent pathways ${ }^{7}$ ). A reciprocal facilitation instead of reciprocal inhibition may even appear ${ }^{8}$. The mechanisms underlying such decreased inhibition below the lesion are still unknown ${ }^{9}$.

GABA and glycine are the primary inhibitory neurotransmitters in the adult spinal cord. The opening of $\mathrm{GABA}_{\mathrm{A}}$ and glycine receptor-gated $\mathrm{Cl}^{-}$channels inhibits neurons as a result of low intracellular $\mathrm{Cl}^{-}$concentration $\left(\left[\mathrm{Cl}^{-}\right]_{\mathrm{i}}\right)$ and hyperpolarized $\mathrm{Cl}^{-}$ equilibrium potential $\left(\mathrm{E}_{\mathrm{Cl}}\right.$; that is, the membrane potential moves away from the action potential threshold). Low $\left[\mathrm{Cl}^{-}\right]_{\mathrm{i}}$ is maintained by the potassium-chloride cotransporter KCC2 (refs. 10,11), which extrudes $\mathrm{Cl}^{-}$. However, in immature neurons, GABA and glycine are depolarizing and often excitatory ${ }^{12}$; the developmental upregulation of KCC2 expression promotes the switch from depolarizing to hyperpolarizing actions of GABA and glycine ${ }^{13,14}$. KCC2 can be downregulated in some pathological conditions ${ }^{11,15-18}$. We now present evidence for a previously unknown mechanism underlying disinhibition and electrophysiological correlates of spasticity after SCI; this mechanism involves a reduction in the expression of KCC2 in the ventral horn, the consequent disruption of $\mathrm{Cl}^{-}$homeostasis and increased excitability of spinal networks and motoneurons.

\section{RESULTS \\ Reduced expression of KCC2 below the SCI}

We analyzed the expression of both the sodium-potassium-chloride cotransporter (NKCC1) and KCC2 by western blotting after SCI caused by spinal thoracic transection and after sham surgery in adult rats. The lumbar protein abundance of NKCC1, which intrudes $\mathrm{Cl}^{-}$, was not significantly affected by SCI (Supplementary Fig. 1). By contrast, KCC2 expression was significantly reduced to $\sim 84 \%$ of control expression within the $7-45 \mathrm{~d}$ after SCI (Fig. 1; $P<0.001$ ). This decrease was progressive (day 1: $92 \% \pm 6 \%$; day 7: $90 \% \pm 5 \%$; day 15: $81 \% \pm 12 \%$; day $45: 78 \% \pm 5 \%)$. In the same samples, we observed no change in the expression of $\beta$-tubulin, used as an internal control (Fig. 1a). KCC2 expression was significantly reduced also after a

\footnotetext{
${ }^{1}$ Laboratoire Plasticité et Physio-Pathologie de la Motricité (UMR6196), Centre National de la Recherche Scientifique (CNRS) \& Aix-Marseille Université, Marseille, France. ${ }^{2}$ Centre de Neurosciences Intégratives et Cognitives, CNRS \& Université de Bordeaux, Talence, France. ${ }^{3}$ Department of Anesthesiology, Vanderbilt University Medical Center, Nashville, Tennessee, USA. ${ }^{4}$ Anesthesiology Research Laboratory, Department of Anesthesiology, University of California-San Diego, La Jolla, California, USA. ${ }^{5}$ Institute of Neurobiology, Slovak Academy of Sciences, Kosice, Slovakia. ${ }^{6}$ Present addresses: Biozentrum, Department of Cell Biology, University of Basel, Basel, Switzerland and Friedrich Miescher Institute for Biomedical Research, Basel, Switzerland (C.J.-X.) and Institut des Neurosciences Cellulaires et Intégratives, UPR3212 CNRS-Université de Strasbourg, Département Nociception et Douleur, Strasbourg, France (P.D.). ${ }^{7}$ These authors contributed equally to this work. Correspondence should be addressed to L.V. (vinay@dpm.cnrs-mrs.fr).
} 
Figure 1 Altered KCC2 expression in lumbar motoneurons after thoracic $\mathrm{SCl}$ in adult rats. (a) Top, western blots of lumbar spinal cords labeled with a KCC2-specific antibody. The 130-140 kDa and the >200 kDa bands correspond to the monomeric ${ }^{14,47,48}$ and oligomeric ${ }^{49}$ proteins, respectively. Bottom, quantification of KCC2 expression in $\mathrm{SCl}$ rats normalized to sham-operated (intact) rats. Data collected from three time points after injury are pooled ( $n=6$ after $7 \mathrm{~d} ; n=4$ after $15 \mathrm{~d}$ and $n=6$ after $45 \mathrm{~d}$ ). $\beta$-tubulin was used as a control ( $n=12$ for each condition). MW, molecular weight. (b-f) Results of immunohistochemical staining with a KCC2specific antibody in lumbar L2-L4 motoneurons of intact and $\mathrm{SCl}$ rats ( $45 \mathrm{~d}$ after injury). (b,c) Single optical sections showing the regular KCC2 staining and the discontinuous clusters of stained spots (arrows) in motoneuron membranes of intact (b) and $\mathrm{SCl}$ (c) rats.

(d,e) Single optical sections showing the density cytoplasmic KCC2-immunolabeled clusters (arrows) in intact (d) and $\mathrm{SCl}$ (e) rats. (f) Left bars, quantification of the density of membrane labeling (ratios of labeled pixel surface per somatic perimeter) in 28 motoneurons from three intact rats and 37 motoneurons from three SCl rats. Right bars, number of cytoplasmic KCC2-immunolabeled clusters per $100 \mu \mathrm{m}^{2}$ of somatic area. $n=24$ and 25 motoneurons in intact and SCl rats, respectively. ${ }^{* * *} P<0.001$, Mann-Whitney test (a) and $t$ test (f). Scale bars, $10 \mu \mathrm{m}$. Error bars on histograms represent s.e.m.

contusion injury, as measured in the chronic phase after $4-5$ months $(P<0.05)$. In transected rats, western blot analysis of the surfacebiotinylated proteins showed that the relative amount of KCC2 included in the membrane where it may be functional, particularly in oligomeric form, was lower compared to intact rats (Supplementary Fig. $2 ; P<0.05)$.

We also analyzed the effect of T9 spinal cord transection at postnatal day 5 (P5). The decrease in KCC2 in the lumbar enlargement $1-3 \mathrm{~d}$ after transection at $\mathrm{P} 5$ was similar to that seen in adults $(88 \pm 3 \%$; $P<0.01$; Fig. 2a).

\section{Reduced targeting of KCC2 to membranes of motoneurons}

We analyzed KCC2 localization by immunohistochemistry in the lumbar spinal cord $45 \mathrm{~d}$ after SCI in adult rats (Fig. 1b-f). KCC2 immunostaining in sham-operated rats was abundant throughout the lumbar segments (data not shown). In the ventral horn, a dense matrix of processes (probably dendrites, as KCC2 is not expressed in axons ${ }^{14}$ ) was stained around large dark cell bodies, presumably motoneurons (Fig. 1d). Neurites stretched out of the gray matter into the lateral and ventral funiculi (data not shown). After SCI, the intensity of KCC2 immunolabeling was reduced mainly in the ventral horn, compared to other spinal areas (Supplementary Fig. 3). At higher magnification, KCC2 immunolabeling in the ventral horn surrounded large cell bodies, suggesting a preferential localization in motoneuron plasma membranes (Fig. 1b). The membrane labeling appeared as a uniform band in intact rats and as clusters in SCI rats (Fig. 1c). The density of labeling (Fig. 1f) and the intensity of staining in the somatic membrane (ratios of mean pixel intensities in motoneurons versus white matter: SCI, $4.29 \pm 0.13, n=204$; shams, $4.86 \pm 0.11, n=204)$ were both significantly lower in SCI rats $(P<0.001)$. After increasing the laser intensity, we observed a diffuse cytoplasmic staining in somas in both groups (Fig. 1d,e). The density of cytoplasmic clusters was, however, higher after SCI (Fig. 1f; $P<0.001$ ). Together, these results suggest that the translocation of KCC2 to the somatic membrane of motoneurons is reduced after SCI.

\section{Increased cell excitability}

Lower expression of KCC2 leads to a higher neuronal $\left[\mathrm{Cl}^{-}\right]_{\mathrm{i}}$ and to $\mathrm{GABA}_{\mathrm{A}}$ receptor-and glycine receptor-mediated depolarization instead of hyperpolarization ${ }^{11,16-18}$. We investigated the physiological correlate of a 10-20\% downregulation of KCC2 (as revealed by western blotting in both adult (Fig. 1a) and P6-P8 rats (Fig. 2a) after SCI) by determining the reversal potential of glycine receptor-and $\mathrm{GABA}_{\mathrm{A}}$ receptor-mediated inhibitory postsynaptic potentials ${ }^{19}\left(\mathrm{E}_{\mathrm{IPSP}}\right)$ in $\mathrm{P} 6-\mathrm{P} 8$ rats transected at $\mathrm{P} 5$. $\mathrm{E}_{\mathrm{IPSP}}$ was depolarized by $\sim 10 \mathrm{mV}$ in SCI rats compared to sham-operated rats (Fig. 2a; $P<0.001)$. Resting membrane potentials $\left(V_{\text {REST }}\right)$ were not affected (Fig. 2a). As a result, $\mathrm{E}_{\mathrm{IPSP}}$ was, on average, below $(1.5 \pm 0.87)$ and above (7.4 2.09) $\mathrm{V}_{\mathrm{REST}}$ in intact and transected rats, respectively.

$\mathrm{E}_{\mathrm{IPSP}}$, which reflects $\mathrm{E}_{\mathrm{Cl}}$, affects the strength of synaptic inhibition $^{20}$. A depolarizing shift of $\mathrm{E}_{\text {IPSP }}$ markedly shortens the functional inhibition through $\mathrm{GABA}_{\mathrm{A}}$ and glycinergic receptors. The action of such inhibitory inputs upon excitatory inputs on the same neuron can even switch from inhibition to facilitation, depending on both $\mathrm{E}_{\mathrm{Cl}}$ and the relative subcellular locations of these inputs. To test whether the $10-\mathrm{mV}$ positive shift of $\mathrm{E}_{\mathrm{IPSP}}$ observed in P6-P8 rats after SCI affects the integrative capability of motoneurons, we used a neuron model $^{20}$ (Fig. 2 b). We generated excitatory postsynaptic potentials (EPSPs) in the soma and IPSPs at various somatic and dendritic locations, first with $\mathrm{E}_{\mathrm{Cl}}$ set to $-75 \mathrm{mV}\left(\mathrm{E}_{\mathrm{Cl}}=\mathrm{V}_{\mathrm{REST}}\right)$ and then with $\mathrm{E}_{\mathrm{Cl}}$ set $10 \mathrm{mV}$ above $\mathrm{V}_{\text {REST }}$ (Fig. 2b-f). We measured the amount of excitatory current required to reach firing threshold (rheobase current) at several times after IPSP onset $(t=0)$. The simulation confirmed that a $10 \mathrm{mV}$ positive shift of $\mathrm{E}_{\mathrm{Cl}}$ markedly reduced the strength of synaptic inhibition (Fig. 2b-d). In addition, $\mathrm{GABA}_{\mathrm{A}}$ and glycinergic inputs located on distal parts of dendrites (100-200 $\mu \mathrm{m}$ away from the soma), which exist in both immature ${ }^{20}$ and mature mammals ${ }^{21}$, may switch their action from inhibition to facilitation after SCI (Fig. 2e,f). We observed similar effects on a neuron model presenting the characteristics of an immature (Fig. 2) or a mature (data not shown) motoneuron (soma diameter: 20 or $40 \mu \mathrm{m}$; input resistance: 47.5 or $2.1 \mathrm{M} \Omega$; dendrite diameter: 2 or $5 \mu \mathrm{m}$, respectively). 
a

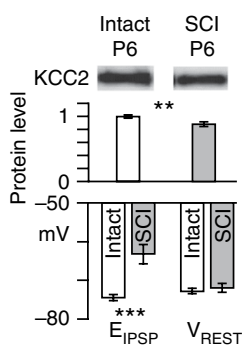

C
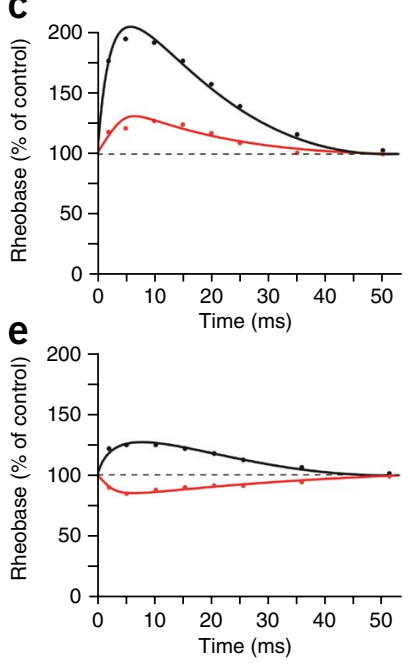

b

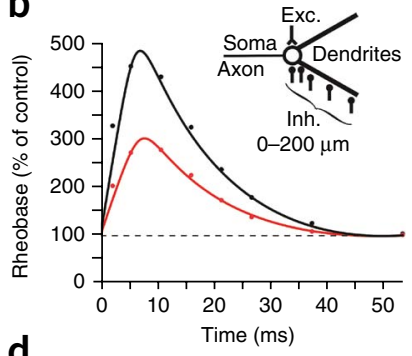

d
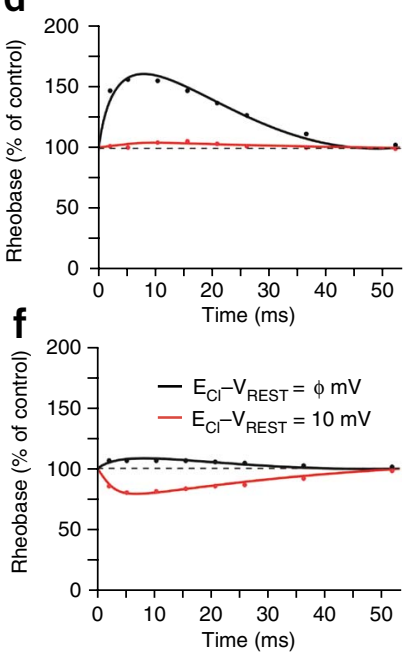

\section{Less KCC2 function increases the excitability of networks}

If hyperreflexia, which is a common feature after SCI, is at least partly due to the decreased expression of KCC2, then a pharmacological blockade of KCC2 in intact rats should have the same effect. To test this hypothesis, we examined the effect of the KCC2 blocker [(dihydroindenyl)oxy] alkanoic acid (DIOA) on spinal reflexes in vitro in neonate rats and in vivo in adult rats. We also used KCC2-deficient mice to assess the effect of reduced expression of KCC2 on spinal reflexes.

In the in vitro spinal cord isolated from neonates, DIOA $(30 \mu \mathrm{M})$ substantially depolarized $\mathrm{E}_{\text {IPSP }}$ without any visible effect on either $V_{\text {REST }}$ or the amplitude of action potentials (Supplementary Fig. 4). Stimulation of a lumbar dorsal root elicits a mixture of excitation and inhibition in ipsilateral and contralateral motoneurons ${ }^{22-24}$. Application of DIOA significantly and reversibly increased polysynaptic responses in both the ipsilateral and the contralateral ventral roots of the same segment $(P<0.001$; Fig. 3$)$.

The Hoffmann reflex is commonly used to assess primary (type Ia) afferents-mediated motoneuronal excitability in individuals suffering from spasticity. Electromyograms typically show two responses,

Figure 3 Blockade of KCC2 with DIOA increases polysynaptic reflexes in the in vitro spinal cord preparation isolated from neonatal rats. (a) Superimposed responses recorded from an L3 ventral root (c L3 VR) after 20 stimulations (one stimulation every $60 \mathrm{~s}$ ) of the contralateral dorsal root (L3 DR) before (control, left) or at the end of the bath application of DIOA $30 \mu \mathrm{M}$ (right). The histograms below the recordings show the number of action potentials evoked by these stimulations (arrows) as a function of time. (b) Time course of the DIOA-induced increase in responses. (c) Mean effects ( \pm s.e.m.) of DIOA or vehicle $(0.2 \%$ DMSO) expressed as percentages of the control responses measured in the last 20 min before drug application in the contralateral and ipsilateral L3 ventral roots $(* * * P<0.001$, Kruskal-Wallis analysis, Dunn's post tests. $n=100$ measurements in five rats for each drug).

Figure 2 Depolarizing shift of $\mathrm{E}_{\mathrm{IPSP}}$ after $\mathrm{SCl}$ and consequences on neuronal excitability. (a) Top, western blotting of KCC2 expression in the lumbar segments $1 \mathrm{~d}$ after $\mathrm{SCl}$ on P5. Middle, quantification of the blots, as normalized to sham-operated (intact) rats. We pooled the data collected from two time periods after injury ( $n=6$ at P6; $n=6$ at P8). Bottom, $\mathrm{E}_{\text {IPSP }}$ and $\mathrm{V}_{\mathrm{REST}}$ in motoneurons $(n=10)$ recorded $1-3 \mathrm{~d}$ after a SCI performed at $\mathrm{P} 5$, or in sham-operated rats $(n=34) . * * P<0.01 ; * * * P<0.001$, Mann- Whitney test. Error bars represent s.e.m. (b-f) Simulation of the effects of $E_{\mathrm{Cl}}$ and location of inhibitory synapses on the excitatory current required to fire an action potential (rheobase current) at the soma (Exc.; b, inset). Graphs show the time course of IPSP-induced changes in the rheobase current $(100 \%=$ rheobase in the absence of inhibitory inputs) for two $E_{C l}$ values (black traces: $E_{C l}=-75 \mathrm{mV}=V_{R E S T}$; red traces: $E_{C l}=-65$ $\mathrm{mV}$ ) and five locations of inhibitory synapses (Inh.; $\mathbf{b}$, inset). A depolarizing shift of $E_{C l}$ markedly reduces the inhibitory action of IPSPs when inhibitory inputs are located on the soma (b) or proximal segments of dendrites (c and d, 25 and $50 \mu \mathrm{m}$ from soma, respectively). Distant synapses (e and f, 100 and $200 \mu \mathrm{m}$ from soma, respectively) can switch their influence on somatic EPSPs from inhibition to facilitation.

an initial $\mathrm{M}$ wave resulting from the direct activation of motor axons and a delayed $\mathrm{H}$ wave resulting from the monosynaptic activation of motoneurons by Ia afferents (Fig. 4a). The $\mathrm{H}$ wave magnitude is normally attenuated by repeated activations at frequencies higher than $0.1 \mathrm{~Hz}$, with a more than $80 \%$ reduction at $5 \mathrm{~Hz}$ in rats $\left(\mathrm{RDD}^{25}\right.$; Fig. 4a). The RDD is progressively reduced in individuals with SCI, and this effect is a reliable correlate of the development of spasticity ${ }^{26}$. We confirmed that the RDD was significantly reduced 4-5 months after a contusive SCI in rats (Supplementary Fig. 5a; $P<0.01$ ). Notably, these rats also showed significantly less KCC2 expression in the lumbar enlargement (Supplementary Fig. 5b; $P<0.05$ ).

We then investigated the effect of blocking KCC2 with $20 \mu \mathrm{g}$ of DIOA, applied directly to the lumbar enlargement through an intrathecal (i.t.) catheter in intact adult rats, while recording the Hoffmann reflex (Fig. 4b-d). The Hoffmann reflex RDD did not significantly decrease with time after i.t. injection of the vehicle DMSO $(0.2 \%$, Fig. $4 c)$. Injection of DIOA did not affect the thresholds, maximal amplitudes and stimulus intensities at which the maximal amplitude is obtained for both $\mathrm{M}$ and $\mathrm{H}$ waves at $0.1-\mathrm{Hz}$ stimulation (Fig. 4a,b and Supplementary Tables 1 and 2). However, an effect of DIOA was clearly present when we used higher frequencies of stimulation, showing that DIOA progressively reduced the RDD (Fig. 4c). Indeed, at $0.5 \mathrm{~Hz}$ and higher frequencies, the amplitude of the
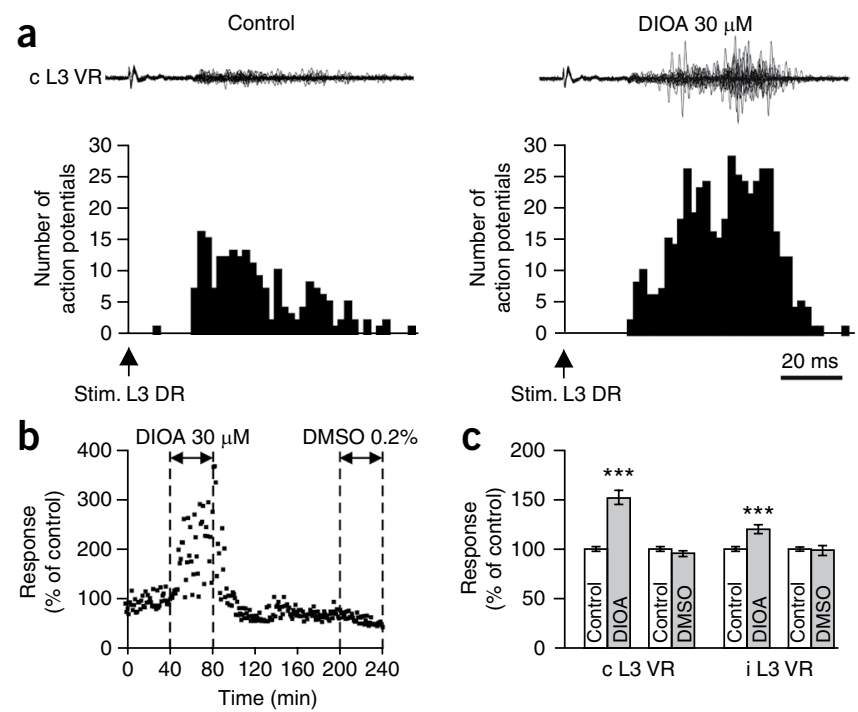
Figure 4 Decreased rate-dependent depression of the Hoffmann reflex after blocking or lowering the expression of KCC2. (a-d) Effects of KCC2 blockade by i.t. injection of DIOA $(20 \mu \mathrm{g})$ in intact rats. $(\mathbf{a}, \mathbf{b})$ Top, $\mathrm{M}$ wave and $\mathrm{H}_{\max }$ responses evoked before (control, a) and 95 min after i.t. injection of DIOA (b). Each trace is the mean response to 17 consecutive stimulations (first three discarded) at 0.1, 0.5, 1,2 or $5 \mathrm{~Hz}$. Bottom, mean relative $\mathrm{H}$ responses to 20 consecutive stimulations at different frequencies in six rats before (left) and after DIOA (right). (c) Time course of the change in $\mathrm{H}$-reflex depression at $0.5 \mathrm{~Hz}$ after i.t. injection of vehicle $(0.2 \%$ DMSO) or DIOA ( $n=102$ measurements in six rats at each time point). The depression decreases progressively after DIOA (Page test, $P<0.05$ ). (d) Mean relative amplitudes of the Hoffmann reflex before (Control) or after DIOA (***P<0.001, KruskalWallis analysis, Dunn's post tests, $n=102$ measurements in six rats at each frequency). (e-g) Decreased RDD in KCC2-deficient mice. (e,f) Mean relative $\mathrm{H}$ responses to 20 stimulations at the indicated frequencies in seven wild-type (WT) and five heterozygous mice with a $\sim 50 \%$ reduction in KCC2 expression a

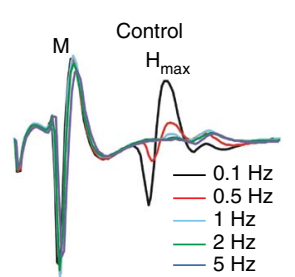

b
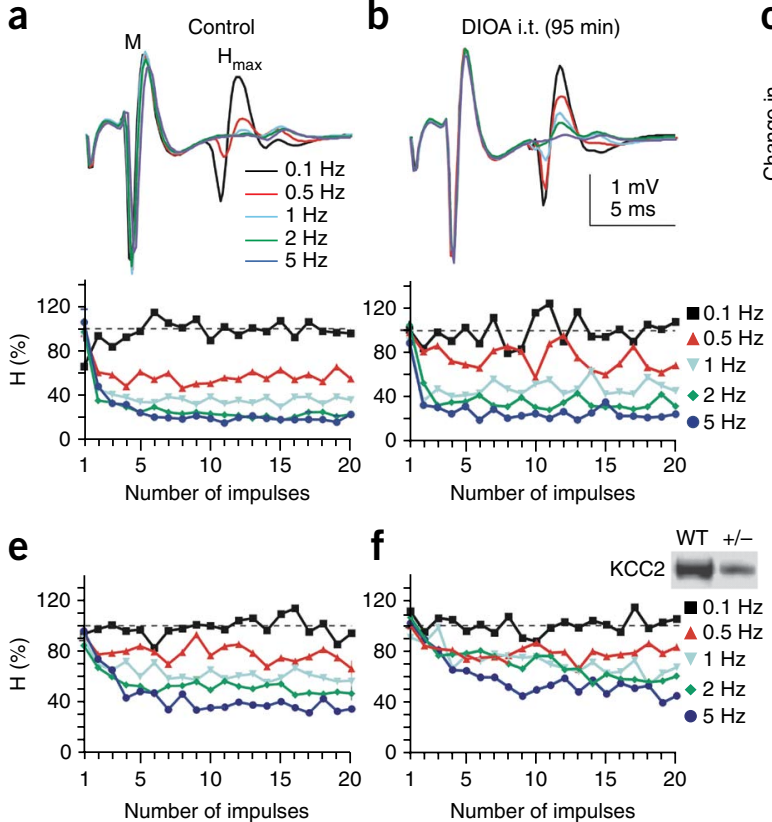

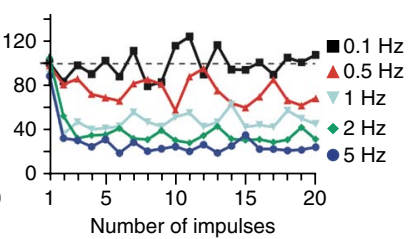

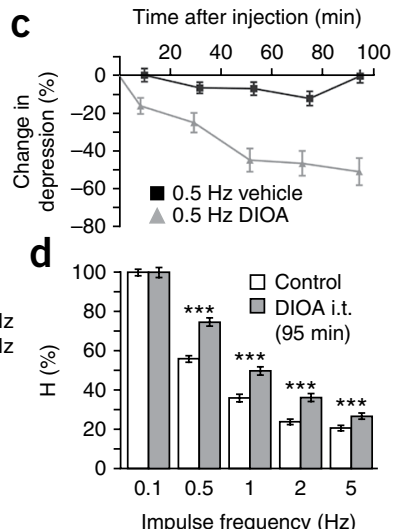

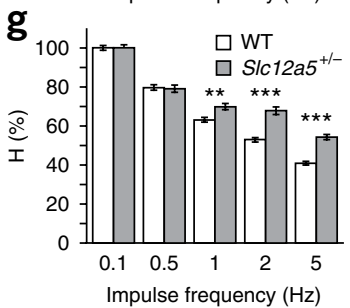
frequency in seven WT and five S/c12a $5^{+/-}$mice, respectively). Error bars on histograms represent s.e.m.

$\mathrm{H}$ wave was larger than it was before blocking KCC2, with a maximal and highly significant effect 95 min after DIOA injection (Fig. 4d; $P<0.001)$. DIOA $(30 \mu \mathrm{M})$ similarly reduced the RDD of the monosynaptic reflex in vitro (Supplementary Fig. 6; $P<0.001$ ).

To confirm, without any pharmacological tool, the role of KCC2 in the regulation of spinal cord reflexes, we used Slc12a5 ${ }^{+/-}$mice $^{27}$ which retain $~ 50 \%$ of normal KCC2 protein amounts in the spinal cord (Fig. 4). RDD was significantly smaller in Slcl2a $25^{+/}$mice compared to wild-type mice (Fig. $4 \mathbf{g} ; P<0.01$ ). The characteristics of the $\mathrm{M}$ and $\mathrm{H}$ waves were not significantly different (Supplementary Table 3 ).

\section{Contribution of BDNF to the downregulation of KCC2}

BDNF, via its receptor TrkB, has been implicated in the downregulation of KCC2 after various neuronal traumas ${ }^{28-30}$. We administered BDNF (10 $\mu$ g i.t.) to the lumbar spinal cord of intact adult rats. This caused a significant reduction in the relative amount of plasmalemmal versus cytoplasmic KCC2 $24 \mathrm{~h}$ later, compared to PBS-injected rats (Fig. 5a; $P<0.05)$. Of note, such a BDNF injection also significantly reduced the Hoffmann reflex RDD $(P<0.01$; Fig. 5b and Supplementary Table 4). We then sought to rescue KCC2 by pretreating rats (30 min before SCI) with the TrkB-Fc chimeric
Figure 5 Interaction between BDNF and KCC2 expression. (a) Relative amount of $\mathrm{KCC} 2$ in the plasma membrane $(\mathrm{mb})$ compared to cytoplasmic compartments in the lumbar spinal cord of intact rats $24 \mathrm{~h}$ after i.t. injection of $10 \mu \mathrm{g}$ BDNF, quantified from western blots. Ratios are normalized to those in PBS-injected rats $\left({ }^{*} P<0.05\right.$ compared to injection of the vehicle PBS, Mann-Whitney test, $n=8$ in each group). (b) Mean relative amplitudes of the Hoffmann reflex in intact rats $24 \mathrm{~h}$ after i.t. injection of BDNF or PBS $(* * P<0.01$; $* * * P<0.001$, one-way ANOVA, Bonferroni's post tests; $n=51$ measurements in three rats for each group). (c) Relative amount of plasmalemmal KCC2, compared to cytoplasmic $\mathrm{KCC} 2$, in the lumbar spinal cord $24 \mathrm{~h}$ after $\mathrm{SCl}$; a single i.t. injection of the TrkB-Fc chimera $(10 \mu \mathrm{g})$ or PBS was performed $30 \mathrm{~min}$ before $\mathrm{SCl}(* * P<0.01$, Mann-Whitney test, $n=10$ in each group). (d) Ratios of the amounts of KCC2 in the plasma membrane and cytoplasmic compartments $15 \mathrm{~d}$ after SCl; BDNF or PBS was injected i.t. $24 \mathrm{~h}$ before western blot analysis of the lumbar spinal cord $\left({ }^{*} P<0.05\right.$ compared to PBS injection, Mann-Whitney test, $n=9$ or 7 rats receiving BDNF or PBS, respectively). (e) Mean relative amplitudes of the Hoffmann reflex at the indicated stimulation frequencies $24 \mathrm{~h}$ after injection of BDNF or PBS in SCI rats $15 \mathrm{~d}$ after injury ${ }^{* * *} P<0.001$, one-way ANOVA, Bonferroni's post tests, $n=85$ and 68 measurements in five BDNF and four PBS-treated rats, respectively). Error bars represent s.e.m.
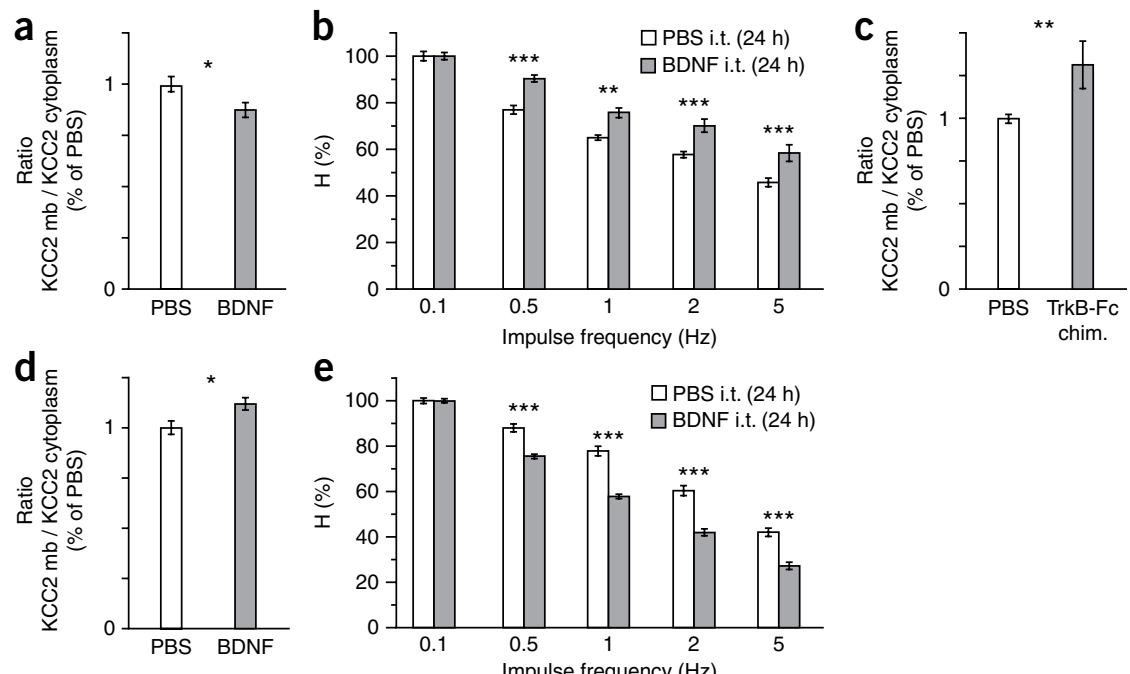
protein, which sequesters endogenously released $\mathrm{BDNF}^{30}$. The relative amount of plasmalemmal versus cytoplasmic KCC2 was significantly larger $24 \mathrm{~h}$ after i.t. lumbar injection of this chimeric protein compared to SCI rats pretreated with PBS (Fig. 5c; $P<0.01$ ).

Although BDNF downregulates KCC2 in intact mature neurons, its effects have been shown to be reversed after axotomy in corticospinal neurons ${ }^{31}$. Similarly, $15 \mathrm{~d}$ after SCI, when hyperreflexia had already developed $^{32}$ (significant reduction of the RDD; $P<0.001$, data not shown), i.t. injection of BDNF increased both the relative amount of plasmalemmal versus cytoplasmic KCC2 (Fig. 5d; $P<0.05)$ and the RDD of the Hoffmann reflex $(P<0.001$; Fig. 5e and Supplementary Table 5) compared to transected rats injected with PBS.

\section{DISCUSSION}

Inhibition is decreased in spinal reflex pathways of individuals with spasticity after $\mathrm{SCI}^{5-9}$. For instance, a cutaneomuscular stimulation that normally elicits a 300-ms EPSP interrupted by a fast IPSP in motoneurons generates a depolarization that lasts for $\sim 1 \mathrm{~s}$ in subjects with chronic $\mathrm{SCI}^{33}$. Considering that inhibitory amino acids are upregulated after complete spinal cord transection ${ }^{34}$ and that the stepping ability on a treadmill of cats with chronic SCI is improved after administration of modest doses of either the glycine receptor antagonist strychnine $\mathrm{e}^{35}$ or the $\mathrm{GABA}_{\mathrm{A}}$ receptor antagonist bicuculline $\mathrm{e}^{36}$, the mechanisms responsible for the reduced inhibition have been misunderstood. The most commonly proposed (though not shown) mechanism to account for the decreased inhibition after SCI is the disruption of a facilitatory supraspinal input onto inhibitory interneurons ${ }^{9,24}$. Our findings provide evidence for a new mechanism that could explain the observed disinhibition in spite of the increased levels of inhibitory amino acids. We show that SCI induces a downregulation of the $\mathrm{Cl}^{-}$extruder KCC2 in the spinal cord below the lesion, particularly in the plasma membrane of motoneurons. SCI might not have exactly the same effects in adult and P5 rats, owing to the not yet fully completed development of the spinal cord and the higher degree of plasticity at P5-P8. However, considering that it is impossible to measure $\mathrm{E}_{\mathrm{IPSP}}$ in adult rat motoneurons because of technical limitations, we used P5-P8 rats in which the switch from depolarizing to hyperpolarizing IPSPs had already occurred ${ }^{19,37}$ and KCC2 expression 1-3 d after SCI is reduced to approximately the same amount as in chronic adults. In this way, we showed that a $10-20 \%$ decrease in KCC2 induces an $\sim 10$ - $\mathrm{mV}$ depolarizing shift of $\mathrm{E}_{\mathrm{IPSP}}$. Such a shift shortens the time window over which IPSPs are able to suppress suprathreshold excitatory inputs ${ }^{20}$. Because the shunting action of IPSPs is local, the inhibitory action of GABA and glycine synapses depends on their location relative to excitatory inputs. Our studies show that synapses located more than $50 \mu \mathrm{m}$ away from the excitation will lose their inhibitory effect after an $\sim 10-\mathrm{mV}$ depolarizing shift of $\mathrm{E}_{\mathrm{Cl}}$. Excitatory actions of GABA and glycine can occur even though the GABA and glycine reversal potentials stay below the action potential threshold. Because the depolarization spreads electrotonically (by contrast to the local shunting action), small depolarizing IPSPs can exert a facilitatory action on distant excitatory inputs such that subthreshold excitations can reach action potential threshold. This may account for the observed reciprocal facilitation - instead of inhibition — of ankle extensors from ankle flexors in individuals with $\mathrm{SCI}^{8}$.

RDD mainly results from the 'postactivation depression' or 'homosynaptic depression' limited to previously activated afferents and has been suggested to be due to a decrease in transmitter release probability following preceding activity ${ }^{38}$. What, then, can account for the tight relationship between RDD and KCC2 expression in our experiments? Stimulation of a peripheral nerve activates not only the monosynaptic connection between Ia afferents and motoneurons but also polysynaptic pathways, some of which are inhibitory to motoneurons in intact animals. When KCC2 expression is low, depolarizing IPSPs can facilitate motoneuron firing via the mechanisms discussed above. In addition, reduced RDD may be related to the role of KCC2 in the buffering of extracellular $\mathrm{K}^{+}\left(\left[\mathrm{K}^{+}\right]_{0}\right)^{39}$. Indeed, repeated stimulation of afferent fibers markedly increases the local $\left[\mathrm{K}^{+}\right]_{\mathrm{o}}$ (ref. 40) so that $\mathrm{K}^{+}$and $\mathrm{Cl}^{-}$transport by $\mathrm{KCC} 2$ can be reversed to a net influx pathway. A lowered expression of KCC2 would then alter the rate of clearance of $\left[\mathrm{K}^{+}\right]_{\mathrm{o}}$, which, in turn, would prolong the depolarization of primary afferent terminals and increase transmitter release, thereby decreasing RDD. Recent observations in the hippocampus of KCC2-depleted mice support this hypothesis ${ }^{41}$.

What are the upstream mechanisms responsible for the downregulation of KCC2 after SCI? Pathways descending from the brainstem influence the expression of KCC2, as suggested by our recent findings that a spinal cord transection at birth prevents both the ontogenic switch from depolarizing to hyperpolarizing IPSPs and the upregulation of KCC2 (ref. 19). We also show here that exogenous BDNF administration alters the expression of plasmalemmal KCC2 in the intact spinal cord of adult rats in a way similar to what is observed after SCI. Notably, BDNF messenger RNA levels are upregulated below the SCI within a short period (6-24 h) after $\mathrm{SCI}^{42}$, and the number of BDNF immunopositive neurons in the ventral horn increases during the first week and returns to control level at $14 \mathrm{~d}^{43}$. This upregulation may trigger the reduction in the expression of KCC2 and account for the rescue effect of pretreatment with the TrkB-Fc chimeric protein. A reversed action of BDNF on KCC2 expression has been described in axotomized cortical neurons ${ }^{31}$. We show that a similar switch also occurs in the spinal cord after SCI (although lumbar motoneurons are not axotomized). Possible intracellular signaling pathways relating BDNF, TrkB receptors and KCC2 expression have been described ${ }^{44}$. Of note, training that improves the recovery of motor function after SCI increases BDNF levels below the injury, and blockade of BDNF prevents the beneficial effects of training. Accordingly, administration of BDNF below a SCI improves spinal learning and 'replaces' the effect of training on locomotor recovery ${ }^{45,46}$. Part of these actions of BDNF may be due to the restoration of endogenous inhibition.

Symptoms of spasticity emerge only several weeks or months after the cord has been damaged. How, then, can the downregulation of KCC2, which starts within the first $24 \mathrm{~h}$ after the SCI, contribute to spasticity? After SCI, motoneurons slowly recover the capacity to show sustained depolarizations (plateau potentials), which result from persistent inward currents (PICs). These PICs are, in part, responsible for long-lasting reflexes and spasms ${ }^{2,3}$. PICs require long depolarizations to fully activate and are inactivated by hyperpolarization. The depolarizing shift of $\mathrm{E}_{\text {IPSP }}$ after KCC2 decrease may enable the long-lasting depolarization evoked by sensory inputs to activate PICs that, then, cannot be turned off because of the lack of hyperpolarization. The downregulation of KCC2 and the upregulation of PICs may thus have a synergistic role in the physiopathology of spasticity.

To conclude, reductions of both inhibition ${ }^{5-9}$ and $\mathrm{RDD}^{26}$ are considered crucial in the pathophysiology of spasticity. The present results demonstrate that a downregulation of KCC2 could contribute to both processes and open new perspectives to prevent or better alleviate spasticity.

\section{METHODS}

Methods and any associated references are available in the online version of the paper at http://www.nature.com/naturemedicine/. 
Note: Supplementary information is available on the Nature Medicine website.

\section{ACKNOWLEDGMENTS}

This study was supported by grants from the Christopher and Dana Reeve Foundation (VB1-0502-2 and VB2-0801-2 to L.V.), the French Agence Nationale pour la Recherche (to L.V.), the French Institut pour la Recherche sur la Moelle épinière et l'Encéphale (to L.V.), the US National Institutes of Health (GM074771 to E.D. and NS051644 to M.M.) and the Slovak Research and Development Agency (APVV-0314-06 to M.M.). We thank the company Pharmaxon for their help and expertise concerning SCI in rats. We also thank all team members, particularly F. Brocard, P. Coulon, K. Sadlaoud and S. Tazerart, for their assistance throughout the experiments, and F. Clarac for his support.

\section{AUTHOR CONTRIBUTIONS}

P.B. designed and performed all in vivo experiments and some in vitro experiments (polysynaptic reflex recordings). S.L. designed and performed the immunohistochemistry, western blot and genotyping experiments. R.B. performed some in vitro experiments (RDD of the monosynaptic reflex and effects of DIOA on motoneurons). H.B. was involved in the confocal analysis and quantification of cell surface expression of KCC2. C.J.-X. contributed to the in vitro studies ( $\mathrm{E}_{\mathrm{IPSP}}$ measurements). C.B., A.S. and P.D. participated in immunohistochemistry, western blot and genotyping experiments and analysis. D.C. designed the cell model used for simulations and participated in the analysis. E.D. generated the mutant mouse and provided valuable advice and expertise for KCC2 regulation. M.M. provided valuable expertise for the in vivo experiments. P.B., S.L. and L.V. wrote the manuscript. L.V. designed and supervised the whole project and contributed to the in vitro experiments, confocal analysis and simulations.

\section{COMPETING INTERESTS STATEMENT}

The authors declare no competing financial interests.

Published online at http://www.nature.com/naturemedicine/.

Reprints and permissions information is available online at http://npg.nature.com/ reprintsandpermissions/

1. Biering-Sørensen, F., Nielsen, J.B. \& Klinge, K. Spasticity-assessment: a review. Spinal Cord 44, 708-722 (2006).

2. Boulenguez, P. \& Vinay, L. Strategies to restore motor functions after spinal cord injury. Curr. Opin. Neurobiol. 19, 587-600 (2009).

3. Li, Y., Gorassini, M.A. \& Bennett, D.J. Role of persistent sodium and calcium currents in motoneuron firing and spasticity in chronic spinal rats. J. Neurophysiol. 91, 767-783 (2004)

4. Katz, R. Presynaptic inhibition in humans: a comparison between normal and spastic patients. J. Physiol. (Paris) 93, 379-385 (1999).

5. Mazzocchio, R. \& Rossi, A. Involvement of spinal recurrent inhibition in spasticity. Further insight into the regulation of Renshaw cell activity. Brain 120, 991-1003 (1997).

6. Boorman, G.I., Lee, R.G., Becker, W.J. \& Windhorst, U.R. Impaired 'natural reciprocal inhibition' in patients with spasticity due to incomplete spinal cord injury. Electroencephalogr. Clin. Neurophysiol. 101, 84-92 (1996).

7. Rémy-Néris, O., Barbeau, H., Daniel, O., Boiteau, F. \& Bussel, B. Effects of intrathecal clonidine injection on spinal reflexes and human locomotion in incomplete paraplegic subjects. Exp. Brain Res. 129, 433-440 (1999).

8. Crone, C., Johnsen, L.L., Biering-Sorensen, F. \& Nielsen, J.B. Appearance of reciprocal facilitation of ankle extensors from ankle flexors in patients with stroke or spinal cord injury. Brain 126, 495-507 (2003).

9. Crone, C. et al. Reduced reciprocal inhibition is seen only in spastic limbs in patients with neurolathyrism. Exp. Brain Res. 181, 193-197 (2007).

10. Delpire, E. \& Mount, D.B. Human and murine phenotypes associated with defects in cation-chloride cotransport. Annu. Rev. Physiol. 64, 803-843 (2002).

11. Payne, J.A., Rivera, C., Voipio, J. \& Kaila, K. Cation-chloride co-transporters in neuronal communication, development and trauma. Trends Neurosci. 26, 199-206 (2003).

12. Ziskind-Conhaim, L. Physiological functions of GABA-induced depolarizations in the developing rat spinal cord. Perspect. Dev. Neurobiol. 5, 279-287 (1998).

13. Rivera, C. et al. The $\mathrm{K}^{+} / \mathrm{Cl}^{-}$co-transporter $\mathrm{KCC} 2$ renders GABA hyperpolarizing during neuronal maturation. Nature 397, 251-255 (1999).

14. Hübner, C.A. et al. Disruption of KCC2 reveals an essential role of K-Cl cotransport already in early synaptic inhibition. Neuron 30, 515-524 (2001).

15. Cohen, I., Navarro, V., Clemenceau, S., Baulac, M. \& Miles, R. On the origin of interictal activity in human temporal lobe epilepsy in vitro. Science 298, 1418-1421 (2002).

16. Coull, J.A. et al. Trans-synaptic shift in anion gradient in spinal lamina I neurons as a mechanism of neuropathic pain. Nature 424, 938-942 (2003).

17. Nabekura, J. et al. Reduction of KCC2 expression and $\mathrm{GABA}_{\mathrm{A}}$ receptor-mediated excitation after in vivo axonal injury. J. Neurosci. 22, 4412-4417 (2002).

18. Toyoda, H. et al. Induction of NMDA and $\mathrm{GABA}_{\mathrm{A}}$ receptor-mediated $\mathrm{Ca}^{2+}$ oscillations with KCC2 mRNA downregulation in injured facial motoneurons. J. Neurophysiol. 89, 1353-1362 (2003).
19. Jean-Xavier, C., Pflieger, J.-F., Liabeuf, S. \& Vinay, L. Inhibitory post-synaptic potentials in lumbar motoneurons remain depolarizing after neonatal spinal cord transection in the rat. J. Neurophysiol. 96, 2274-2281 (2006).

20. Jean-Xavier, C., Mentis, G.Z., O'Donovan, M., Cattaert, D. \& Vinay, L. Dual personality of GABA/glycine-mediated depolarizations in the immature spinal cord. Proc. Natl. Acad. Sci. USA 104, 11477-11482 (2007).

21. Alvarez, F.J., Dewey, D.E., Harrington, D.A. \& Fyffe, R.E. Cell-type specific organization of glycine receptor clusters in the mammalian spinal cord. J. Comp. Neurol. 379, 150-170 (1997).

22. Jiang, Z., Carlin, K.P. \& Brownstone, R.M. An in vitro functionally mature mouse spinal cord preparation for the study of spinal motor networks. Brain Res. 816, 493-499 (1999).

23. Wallis, D.I. \& Wu, J. Fast and slow ipsilateral and contralateral spinal reflexes in the neonate rat are modulated by 5-HT. Gen. Pharmacol. 23, 1035-1044 (1992).

24. Edgley, S.A. \& Aggelopoulos, N.C. Short latency crossed inhibitory reflex actions evoked from cutaneous afferents. Exp. Brain Res. 171, 541-550 (2006).

25. Thompson, F.J., Reier, P.J., Lucas, C.C. \& Parmer, R. Altered patterns of reflex excitability subsequent to contusion injury of the rat spinal cord. J. Neurophysiol. 68, 1473-1486 (1992)

26. Grey, M.J. et al. Post-activation depression of Soleus stretch reflexes in healthy and spastic humans. Exp. Brain Res. 185, 189-197 (2008)

27. Woo, N.S. et al. Hyperexcitability and epilepsy associated with disruption of the mouse neuronal-specific K-Cl cotransporter gene. Hippocampus 12, 258-268 (2002).

28. Coull, J.A. et al. BDNF from microglia causes the shift in neuronal anion gradient underlying neuropathic pain. Nature 438, 1017-1021 (2005).

29. Rivera, C. et al. BDNF-induced TrkB activation down-regulates the $\mathrm{K}^{+}-\mathrm{Cl}^{-}$ cotransporter KCC2 and impairs neuronal $\mathrm{Cl}^{-}$extrusion. J. Cell Biol. 159, 747-752 (2002).

30. Miletic, G. \& Miletic, V. Loose ligation of the sciatic nerve is associated with TrkB receptor-dependent decreases in KCC2 protein levels in the ipsilateral spinal dorsal horn. Pain 137, 532-539 (2008).

31. Shulga, A. et al. Posttraumatic $\mathrm{GABA}_{A}$-mediated $\left[\mathrm{Ca}^{2+}\right] \mathrm{i}$ increase is essential for the induction of brain-derived neurotrophic factor-dependent survival of mature central neurons. J. Neurosci. 28, 6996-7005 (2008).

32. Yates, C. et al. The onset of hyperreflexia in the rat following complete spinal cord transection. Spinal Cord 46, 798-803 (2008).

33. Norton, J.A., Bennett, D.J., Knash, M.E., Murray, K.C. \& Gorassini, M.A. Changes in sensory-evoked synaptic activation of motoneurons after spinal cord injury in man. Brain 131, 1478-1491 (2008).

34. Edgerton, V.R., Tillakaratne, N.J., Bigbee, A.J., De Leon, R.D. \& Roy, R.R. Plasticity of the spinal neural circuitry after injury. Annu. Rev. Neurosci. 27, 145-167 (2004).

35. de Leon, R.D., Tamaki, H., Hodgson, J.A., Roy, R.R. \& Edgerton, V.R. Hindlimb locomotor and postural training modulates glycinergic inhibition in the spinal cord of the adult spinal cat. J. Neurophysiol. 82, 359-369 (1999).

36. Edgerton, V.R. et al. Use-dependent plasticity in spinal stepping and standing. Adv. Neurol. 72, 233-247 (1997).

37. Stil, A. et al. Developmental up-regulation of the potassium-chloride cotransporter type 2 in the rat lumbar spinal cord. Neuroscience 164, 809-821 (2009).

38. Hultborn, $\mathrm{H}$. et al. On the mechanism of the post-activation depression of the H-reflex in human subjects. Exp. Brain Res. 108, 450-462 (1996).

39. Payne, J.A. Functional characterization of the neuronal-specific $\mathrm{K}-\mathrm{Cl}$ cotransporter implications for $\left[\mathrm{K}^{+}\right]$o regulation. Am. J. Physiol. 273, C1516-C1525 (1997).

40. Syková, E., Jendelova, P., Svoboda, J. \& Chvatal, A. Extracellular $\mathrm{K}^{+}, \mathrm{pH}$ and volume changes in spinal cord of adult rats and during postnatal development. Can. J. Physiol. Pharmacol. 70 Suppl, S301-S309 (1992).

41. Riekki, R. et al. Altered synaptic transmission but normal long-term plasticity in mice lacking hyperpolarizing $\mathrm{GABA}_{\mathrm{A}} \mathrm{R}$-mediated inhibition in $\mathrm{CA1}$ pyramidal neurons. J. Neurophysiol. 99, 3075-3089 (2008).

42. Nakamura, M. \& Bregman, B.S. Differences in neurotrophic factor gene expression profiles between neonate and adult rat spinal cord after injury. Exp. Neurol. 169, 407-415 (2001).

43. Li, X.L. et al. Temporal changes in the expression of some neurotrophins in spinal cord transected adult rats. Neuropeptides 41, 135-143 (2007).

44. Rivera, C. et al. Mechanism of activity-dependent downregulation of the neuronspecific K-Cl cotransporter KCC2. J. Neurosci. 24, 4683-4691 (2004).

45. Gómez-Pinilla, F. et al. BDNF and learning: evidence that instrumental training promotes learning within the spinal cord by up-regulating BDNF expression. Neuroscience 148, 893-906 (2007).

46. Boyce, V.S., Tumolo, M., Fischer, I., Murray, M. \& Lemay, M.A. Neurotrophic factors promote and enhance locomotor recovery in untrained spinalized cats. J. Neurophysiol. 98, 1988-1996 (2007).

47. Lu, J., Karadsheh, M. \& Delpire, E. Developmental regulation of the neuronalspecific isoform of $\mathrm{K}-\mathrm{Cl}$ cotransporter $\mathrm{KCC} 2$ in postnatal rat brains. J. Neurobiol. 39, 558-568 (1999).

48. Williams, J.R., Sharp, J.W., Kumari, V.G., Wilson, M. \& Payne, J.A. The neuronspecific $\mathrm{K}-\mathrm{Cl}$ cotransporter, $\mathrm{KCC} 2$. Antibody development and initial characterization of the protein. J. Biol. Chem. 274, 12656-12664 (1999).

49. Blaesse, P. et al. Oligomerization of KCC2 correlates with development of inhibitory neurotransmission. J. Neurosci. 26, 10407-10419 (2006). 


\section{ONLINE METHODS}

Animals. Generation and genotyping of the transgenic mice used have been

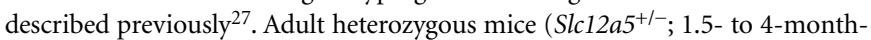
old males) express about $50 \%$ of KCC2 compared to the corresponding wildtype C57BL/6 background mice. We housed mice and Wistar rats (Charles River Laboratories and home bred) in a temperature-controlled animal-care facility with a $12 \mathrm{~h}$ light-dark cycle. We made all efforts to minimize animal suffering and the number of animals used. We performed experiments in accordance with French regulations (Ministry of Food, Agriculture and Fisheries; Division of Health and Protection of Animals). The local Direction of Veterinary Services (Direction départementale des Services Vétérinaires des Bouches du Rhône) and ethical committee (Comité d'éthique de Marseille pour l'expérimentation animale) delivered the appropriate licenses and approved the protocols, respectively.

Spinal cord injury and implantation of intrathecal catheters. We anesthetized female rats (220-280 g) with a mixture of ketamine (Imalgene, Merial, $80 \mathrm{mg}$ per kg body weight, intraperitoneally) and xylazine (Rompun, Bayer, $10 \mathrm{mg}$ per kg body weight, intraperitoneally) and neonates by hypothermia. After laminectomy, we transected the spinal cord at the thoracic level (T8-T10; in P5 rats, we removed one segment of the cord). We treated sham-operated rats in the same way except for the spinal cord transection. Post-surgical treatments and care are described in Supplementary Methods.

For i.t. drug delivery, we inserted PE- 5 catheters (outside diameter: $0.36 \mathrm{~mm}$ ) under the dura, either through the cisternal membrane (catheter length: $7.2 \mathrm{~cm}$ ) or with a caudal approach from L2 vertebra (catheter length: $1.3 \mathrm{~cm}$ ), toward the lumbar enlargement (L2-L5) located mainly under the T13 vertebra. We delivered $20 \mu \mathrm{l}$ of drugs or vehicle (0.1 M PBS, or for DIOA 0.2\% DMSO plus $0.9 \% \mathrm{NaCl}$ buffered to $\mathrm{pH} 7.4$ with PBS) followed by a $6-\mu \mathrm{l}$ flush (dead volume of the catheter) in $1.5 \mathrm{~min}$. We checked the precise placement of catheters postmortem.

Western blots. We killed animals by decapitation after isoflurane anesthesia and quickly dissected out and froze the L1-L5 parts of the spinal cords. We homogenized samples in cold lysis buffer A (modified RIPA buffer, Supplementary Methods) and centrifuged samples at $18,000 \mathrm{~g}$ for $30 \mathrm{~min}$ at $4{ }^{\circ} \mathrm{C}$. We determined supernatant protein concentrations with a detergent-compatible (DC) protein assay (Bio-Rad). For cytoplasmic and membrane fractions, we homogenized samples in cold lysis buffer B (Supplementary Methods) without detergent and centrifuged them first at 7,000 $\mathrm{g}$ for $5 \mathrm{~min}$ and then at $29,700 \mathrm{~g}$ for $1.5 \mathrm{~h}$ at $4{ }^{\circ} \mathrm{C}$. We collected pellets in lysis buffer A without DTT (membrane-enriched fractions) and supernatants (cytoplasmic fractions). We then immunoprecipitated KCC2 (Supplementary Methods), separated samples in 7\% SDS-PAGE and transferred them to a polyvinylidene fluoride membrane. After blockade in Trisbuffered saline plus 5\% nonfat dry milk, we exposed membranes overnight at $4{ }^{\circ} \mathrm{C}$ to a polyclonal rabbit KCC2-specific antibody diluted 1 in 500 (Millipore) in the blocking solution. We used an ImmunoPure goat horseradish peroxidaseconjugated rabbit-specific antibody $\left(1\right.$ in 500 in blocking solution, $1 \mathrm{~h}$ at $22^{\circ} \mathrm{C}$ ) for chemiluminescent detection (Pierce Biotech). We measured signal intensities with the image analysis software Quantity-One (Bio-Rad).

Immunohistochemistry. We killed adult rats $45 \mathrm{~d}$ after sham surgery or SCI ( $n=3$ in each group). After intracardiac perfusion and post-fixation in $4 \%$ paraformaldehyde, we cut lumbar segments (30- $\mu \mathrm{m}$-thick sections) with a vibratome. We mounted sections from the six rats on the same slides and processed them simultaneously. We permeated sections for $60 \mathrm{~min}(0.2 \%$ Triton X-100) and preincubated them for $45 \mathrm{~min}$ at $22^{\circ} \mathrm{C}$ in PBS containing 3\% BSA plus normal goat serum diluted 1 in 100 . We incubated sections overnight at $22^{\circ} \mathrm{C}$ in an affinity-purified rabbit KCC2-specific polyclonal antibody (1 in 200; Millipore). We then revealed the labeling with a goat Alexa Fluor 546-conjugated rabbit-specific antibody ( 1 in $400,1 \mathrm{~h}$ at $22^{\circ} \mathrm{C}$, Molecular Probes) and mounted cover slips with a gelatinous aqueous medium. We scanned all motoneurons of the ventral horn with visible nuclei using a confocal microscope (Olympus FV500, Zeiss LSM 510 META) at ×63 magnification and digitized stacks of $1-\mu \mathrm{m}$-thick optical sections. Quantification is presented in the Supplementary Methods.

In vitro electrophysiological recordings. After hypothermic anesthesia, we dissected out the spinal cords (sacral segments up to T8-T10) and L3-L5 dorsal and ventral roots of newborn rats. We performed dissections and electrophysiological procedures under continuous perfusion with an oxygenated artificial cerebrospinal fluid (composition available in the Supplementary Methods). Measurements of $\mathrm{E}_{\mathrm{IPSP}}$ and reflex recordings are described in Supplementary Methods.

Simulations. We simulated interactions between IPSPs and EPSPs at various $\mathrm{E}_{\mathrm{Cl}}$ values with a recently described compartment mode ${ }^{20}$ (Supplementary Methods).

In vivo electrophysiological recordings. We measured the Hoffmann reflex in adult rats under ketamine anesthesia (100 mg per kg body weight, intramuscularly) with a pair of stainless steel needle electrodes transcutaneously inserted into the vicinity of the tibial nerve for stimulation. We placed the recording electrode into the plantar muscle beneath the ankle and the reference electrode subcutaneously into the foot. RDD measurement is described in Supplementary Methods.

Statistical analyses. We expressed group measurements as means \pm s.e.m. We used $t$ tests to compare two groups and one-way ANOVA to compare more than two groups, or we used corresponding nonparametric analyses when data were not normally distributed or when we analyzed small samples $(<20)$ (Graphpad Prism 4 software). We analyzed the effect of time after DIOA i.t. injection by testing the hypothesis of a progressive diminution of RDD with time using the Page test (StatXact.7). The level of significance was set at $P<0.05$.

Additional methods. Detailed methodology is described in the Supplementary Methods. 
Copyright of Nature Medicine is the property of Nature Publishing Group and its content may not be copied or emailed to multiple sites or posted to a listserv without the copyright holder's express written permission.

However, users may print, download, or email articles for individual use. 